\title{
On the stability of continuously stratified quasi-geostrophic hetons
}

\author{
Jean N. Reinaud ${ }^{1} \ddagger$ \\ ${ }^{1}$ Mathematical Institute, North Haugh, St. Andrews, KY169SS, Scotland
}

\begin{abstract}
In this paper we examine the stability of quasi-geostrophic hetons in a stably, continuously stratified fluid. To this purpose we first determinate numerically equilibrium states. Equilibrium hetons consist of two vortices of equal and opposite strength lying at different depths that are steadily translating without deforming. The situation is studied through a parameter space comprising the vertical offset between the vortices, their horizontal separation distance and their aspect ratio. The study first shows that the equilibrium vortices are not only strongly deformed in the vertical but that their instability modes are also varying within the height of the structures. The main purpose of the present contribution is to study families of equilibria which stem from the case of two vertically aligned cylindrical vortices. It is however shown that other branches of solutions exist with different properties. The paper concludes that hetons may be sensitive to baroclinic instabilities provided the separation distance between the poles of the hetons is moderate both in the horizontal and in the vertical directions. The hetons become stable and efficient ways to transport properties as fas as the poles are distant from one another. The critical separation distance in a non-trivial function of the radius-to-height aspect ratio of the poles.
\end{abstract}

Keywords: Vortex Dynamics, Vortex equilibria, Baroclinic instability, Hetons

$\ddagger$ Corresponding author: jean.reinaud@st-andrews.ac.uk 


\section{Introduction}

Vortices play an essential part of the oceans and the atmosphere dynamics. Ebbesmayer et al (1986) estimated that the surface of the North Atlantic alone was populated at all time by about 1000 up to 10000 vortices. Vortices are not restricted to the surface layers of the oceans and also exist at various depths. These vortices may arise for example from the baroclinic-barotropic destabilisation of intense jets such as the Gulf Stream. Such vortices (called 'rings') have been extensively studied in the literature, see a review by Robinson (1983).

In such contexts, vortices can be seen as compact volumes of anomalous potential vorticity. Potential Vorticity is the central dynamical prognostic variable for stably stratified, rapidly rotating flows, as observed at large and meso scales in the oceans and the atmosphere. Indeed, within the quasi-geostrophic approximation, all fields derive from the potential vorticity (hereinafter referred to as PV), see e.g. Vallis (2006).

The stability and the dynamics of pairs of three dimensional geophysical vortices have been extensively studies. Both pairs of co-rotating (von Hardenberg et al (2000), Dritschel (2002), Reinaud \& Dristchel (2002), Bambrey, Reinaud \& Dritschel (2007), Ozugurlu, Reinaud \& Dritschel (2008) and counter-rotating (see e.g. Reinaud \& Dritschel (2009)) have been considered in the literature. In all these studies the two vortices were either horizontally aligned or at least overlapped in the vertical. In the present paper we address the stability of a pair of counter rotating vortices in the case where the two vortices are completely offset in the vertical, i.e. they do not share any vertical level. Due the lack of vertical advection within the quasi-geostrophic approximation, the vortices are physically prohibited to enter into contact. However the configuration is still sensitive to the baroclinic instability due to the vertical shear vortices induce to one another (and to some extend on themselves). Such vortex configurations are referred to as 'Hetons', following the seminal paper by Hogg \& Stommel (1985). Note that the motion of vortex charges (i.e. singularities) both in the continuous approximation and in a two-layer model has been originally studied by Gryanik (1983a) (resp. 1983b) as well as in Young (1985) and Gryanik and Tevs (1989) in a N-layer model. Experimental measurement are provided for a two layer flow in Griffiths and Hopfinger (1986). The translation velocity of hetons can be found in these papers. Finite core hetons have mostly been studied in layered models. For example, Flierl (1988) studied the stability of hetons in a two layer model. In this study the two vortices were placed on the top of one another. Reviews of the topic in layered models may be found in Gryanik, Sokolovskiy and Verron (2006) and Sokolovskiy and Verron (2014). Sokolovskiy (1997) studied the stability of hetons in a two layer model with finite amplitude potential vorticity in the middle layer. Both studies showed the influence of the aspect ratio of the vortices, wider vortices being sensitive to higher azimuthal modes. Reinaud \& Carton (2009) studied the stability and nonlinear evolution of vertically aligned hetons using both a three layer model and a discretised model based on continuous stratification. In their study no potential vorticity is placed between the 
poles of the heton (i.e. no PV in the middle layer for the three layer model). This study confirmed the sensitivity to higher azimuthal modes of the wider vortices, as well as showing a weakening of the instabilities and their shifting to lower azimuthal modes as the poles of the hetons are separated in the vertical. In this study vortex equilibria could be found in an ad hoc manner, due to the lack of horizontal shift between the two poles. In that case, one can assume a horizontal radial form for the poles. i.e. the overall $\mathrm{PV}$ distribution is of the form $q(r, z)$ where $r$ is the cylindrical polar radius and $z$ the height. In that case one can find a solution for the stream function of the form $\psi(r, z)$. This means that the streamlines for the (horizontal) advecting velocity field are circular. Therefore they coincide with the boundary of the vortices implicitly set by the $\mathrm{PV}$ distribution $q(r, z)$. In that case the boundary of the vortex do not deform as it is tangent to the velocity field. However the two poles do not induce a global velocity field on one another and the structure stands still (while rotating on itself).

In the present study the poles are no longer aligned in the vertical and there is no trivial equilibrium shape for the vortices. They first need to be computed. Once the equilibria are determined, we perform a linear stability analysis. The study can be completed by the computation of the nonlinear evolution of unstable equilibria using a purely Lagrangian method: Contour Surgery, see Dritschel (1989) for the twodimensional method and e.g. Reinaud \& Dritschel (2009) for its application to threedimensional QG model.

We find that the instabilities first observed for no horizontal offset disappear rather rapidly as the vortices are separated in the horizontal. More to this, we show that the vertical structure of the instability modes is crucial to the dynamics. Finally we shortly illustrate the existence of multiple branches of possible equilibria with different stability properties.

\section{The numerical model and basic equations}

The equations governing a continuously stratified and rapidly rotating flow within the quasi-geostrophic (QG) approximation are first presented. For the sake of simplicity, we use the QG equations derived from the Boussinesq approximation, and we take the buoyancy frequency $N$ constant. Within the Boussinesq approximation, this means we assume a linear background stratification of the density. We also take the Coriolis frequency $f$ constant, neglecting its background latitudinal variation. These two simplifying assumptions allow to rescale the problem in the vertical direction by the ratio $N / f$, and the linear relation between the stream function $\psi$ and the potential vorticity anomaly $q$ is a Poisson's equation. The full governing equations read

$$
\begin{aligned}
& \frac{\mathrm{d} q}{\mathrm{~d} t}=0, \\
& \Delta \psi=q, \\
& u=-\frac{\partial \psi}{\partial y}, \text { and } v=\frac{\partial \psi}{\partial x},
\end{aligned}
$$


where $\mathrm{d} / \mathrm{d} t=\partial / \partial t+u \partial / \partial x+v \partial / \partial y$ is the material derivative, $(u, v)$ is the advecting (geostrophic) velocity.

Equation (2) can be inverted using the appropriate Green's function and the $\psi(x, y, z) \rightarrow 0$ as $x, y, z \rightarrow \infty$ for a compact support of potential vorticity.

$$
\psi(\mathbf{x})=\iiint G\left(\mathbf{x}-\mathbf{x}^{\prime}\right) q\left(\mathbf{x}^{\prime}\right) \mathrm{d} \mathbf{x}^{\prime} ; \quad G(\mathbf{x})=-\frac{1}{4 \pi|\mathbf{x}|}
$$

and $\mathbf{x}=(x, y, z)$.

The fluid domain is discretised in the vertical by $N$ layers. To calculate the influence of the PV distribution laying on the layer $k$, we first integrate the Green function over the thickness $d z$ of the layer. This is done analytically

$$
\begin{aligned}
& G_{k}(x, y, z)=\int_{z_{k}-d z / 2}^{z_{k}+d z / 2} G\left(x, y, z-z^{\prime}\right) \mathrm{d} z^{\prime} \\
& =-\frac{1}{4 \pi} \ln \frac{z-\left(z_{k}-d z / 2\right)+\sqrt{r_{h}^{2}+\left(z-\left(z_{k}-d z / 2\right)\right)^{2}}}{z-\left(z_{k}+d z / 2\right)+\sqrt{r_{h}^{2}+\left(z-\left(z_{k}+d z / 2\right)\right)^{2}}} .
\end{aligned}
$$

where $r_{h}^{2}=x^{2}+y^{2}, z_{k}$ is the mid-depth of the layer and $d z$ is its thickness. We obtain

$$
\psi=\sum_{k=1}^{N} \iint_{S} q_{k}(x, y) G_{k}\left(x-x^{\prime}, y-y^{\prime}, z\right) \mathrm{d} x^{\prime} \mathrm{d} y^{\prime} .
$$

Then we further integrate the function to be transformed into contour integral using Green's theorem

$$
P=-\frac{1}{2} \int G_{k}\left(x-x^{\prime}, y-y^{\prime}, z\right) \mathrm{d} y^{\prime}, \quad Q=\frac{1}{2} \int G_{k}\left(x-x^{\prime}, y-y^{\prime}, z\right) \mathrm{d} x^{\prime},
$$

and,

$$
\iint_{\mathcal{S}} G_{k} \mathrm{~d} S=\oint_{\mathcal{C}} P \mathrm{~d} x+Q \mathrm{~d} y
$$

Both $P$ and $Q$ are obtained analytically.

The method used to find the equilibrium adapted from the ones outlined in Reinaud \& Dritschel (2002) with the rotation of the pair of vortices being replaced by their translation. It consists of an iterative method which makes the vortex contours converge to streamlines, making use explicitly of the integrals presented above. The method used to perform the linear stability is the same as used in Reinaud and Dritschel (2002) and originate from the two-dimensional method proposed in Pierrehumbert (1980). The modes of instability correspond to deformation modes of the boundary of the vortices. Note that this framework allows to study a flow explicitly unbounded. Due to the inherent existence of small amplitude numerical truncation error, and the finite numerical accuracy of the various schemes (including the one used to calculate the equilibria, which leads to a very small amount of residual unsteadiness in the states), any mode with a growth rate less that $2.5 e-3$ is considered stable. 


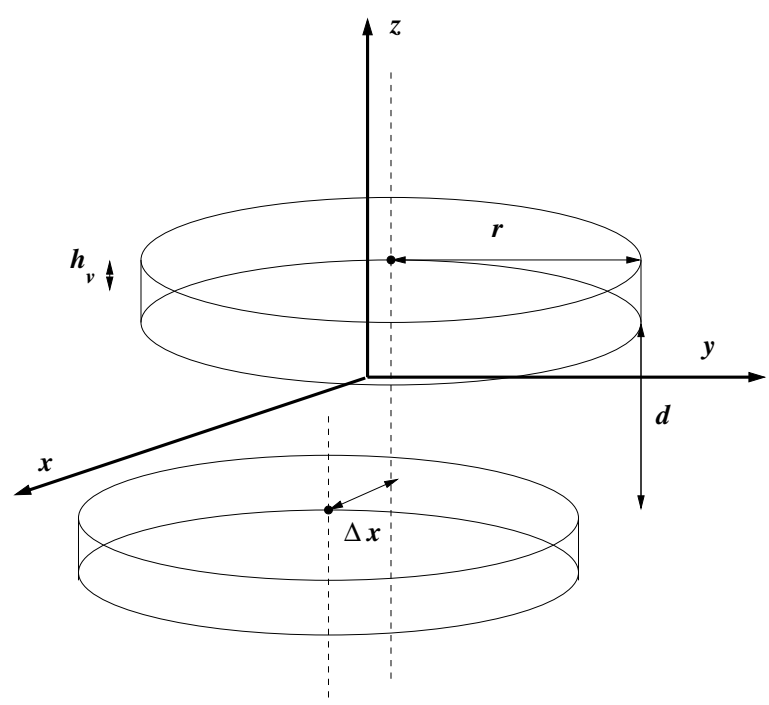

Figure 1. General geometry of a finite core heton and definition of the main symbols. The notations are the same as in Reinaud and Carton (2009). $r$ is the radius of the poles, $h_{v}$ is the half-height of the pole. $d$ is the vertical separation between the poles. $\Delta x$ is the horizontal separation between the poles centres.

\section{General geometries}

The general geometry of the configuration is presented in figure 1. Each pole (vortex) of the heton has an identical volume, and an assigned PV of $\pm 2 \pi$. They are characterised by a half-height $h_{v}$ and a mean horizontal radius $r$ (the surface area of each horizontal cross-section of the vortices is $\pi r^{2}$ and the poles have a volume of $2 h_{v} r^{2} \pi$ each). Initially (for no horizontal offset) the pole are indeed two cylinders of radius $r$ and half-height $h_{v}$. The $x$-axis is taken to be the axis joining the two centres without loss of generality. $\Delta x$ represents the horizontal separation distance between the centres of the two poles of the heton. In this paper we also make use of $\delta$ the gap between the two outermost edges of the vortices. Initially $\delta=\Delta x+2 r=2 r$. The gap $\delta$ contains information on the centroid separation between the poles as well as their width, known to be important. $d$ corresponds to the vertical distance without PV in-between the two poles (in an analogue 3-layer model that would be the thickness of the middle layer). In the numerical approach, each vortex is mapped in the vertical by $n_{v}=25$ layers. The horizontal resolution is chosen such that it is consistent with the vertical one. If $n_{v}$ contours are mapping the height $2 h_{v}$ of the cylindrical poles then the vertical resolution is $d z=2 h_{v} / n_{v}$. Therefore the number of points needed to map a circular horizontal contour of length $2 \pi r$ is $n_{p} \simeq 2 \pi r / d z \simeq \pi\left(r / h_{v}\right) n_{v}$. This set-up provides a very high resolution of the vortices. 


\section{Equilibria}

\subsection{First family of symmetric equilibria}

The vortices of the heton share the same volume and the same PV (in absolute value). Moreover the equations themselves are symmetric ( $x$ and $y$ play similar roles and $z$ can be reversed). As a consequence, it is natural to first investigate branches of symmetric solutions where the vortices are symmetric with respect to the centre of the domain. In the discrete problem this means in practice that contours defining the horizontal crosssection of the vortices are first symmetric with respect to the $x$-axis which joins the two centres. Furthermore the $j^{\text {th }}$ horizontal contour of the first vortex is the mirror image, with respect to the plane $x=0$, of the $n_{v}-(j-1)^{t h}$ contour of the second vortex, where $n_{v}$ is the number of contour spanning each vortex.

Since we know that a radial distribution of $\mathrm{PV}$ is an equilibrium, we start the calculation with two cylinders of PV vertically aligned. We then offset them slightly in the horizontal direction and find the equilibrium for this new separation distance. The initial guess in the iterative method consists of the previous equilibrium shape obtained, the vortices being only slightly pushed further apart. This procedure is resumed until the separation between the two centroid exceeds twice the initial mean radius or when the algorithm fails to converge. In the later case we can restart the calculation from further apart. Indeed when further apart the mutual influence of the two vortices decreases and near-to-circular horizontal shapes should be at equilibrium as well. The algorithm is then resumed and we attempt to complete the branch of solutions by decreasing the separation between the two vortices of the family of equilibria sought. In certain cases however, we cannot complete the branch and our data are incomplete for such branches.

The data presented in this section relate to branches of solutions stemming from the initial radial distribution of PV for no horizontal separation. We will mention in a subsequent section the existence of other branches of solutions.

Figure 2 illustrates the shape of the vortices at equilibria for 5 examples taken along the branch of solutions for $r / h_{v}=4$ and $d / h_{v}=1$. The shapes are in fact qualitatively generic for this family of solutions for the range of aspect ratios $r / h_{v}$ and vertical offsets $d / h_{v}$ sought. For small horizontal offsets, the equilibrium remain close to the cylindrical one. The deformation increases first as the separation is increased. It then reaches a maximum. As the distance is further increased the deformation decreases as the mutual influence is then reduced. Qualitatively, in a point vortex approximation, the horizontal velocity induced by one pole varies as $\ell /\left(\ell^{2}+z^{2}\right)^{3 / 2}$ hence the vertical shear as $-3 \ell z /\left(\ell^{2}+z^{2}\right)^{5 / 2}$ where $\ell$ (resp. $z$ ) is a horizontal (resp. vertical) distance. The shear reaches a maximum at finite distance $(\ell= \pm z / 2)$. It is expected that the deformation needed to withstand the vertical shear will increase with its intensity. Note that similar qualitative arguments can be put forward for the horizontal shear.

We next illustrate the shape of the vortices at equilibrium along the full branch of equilibria (spanned by the gap $\delta$ ) for $r / h_{v}=4$ and various values of the vertical offset $d / h_{v}$. We focus in particular on the vertical dependence of the shape. This shape 

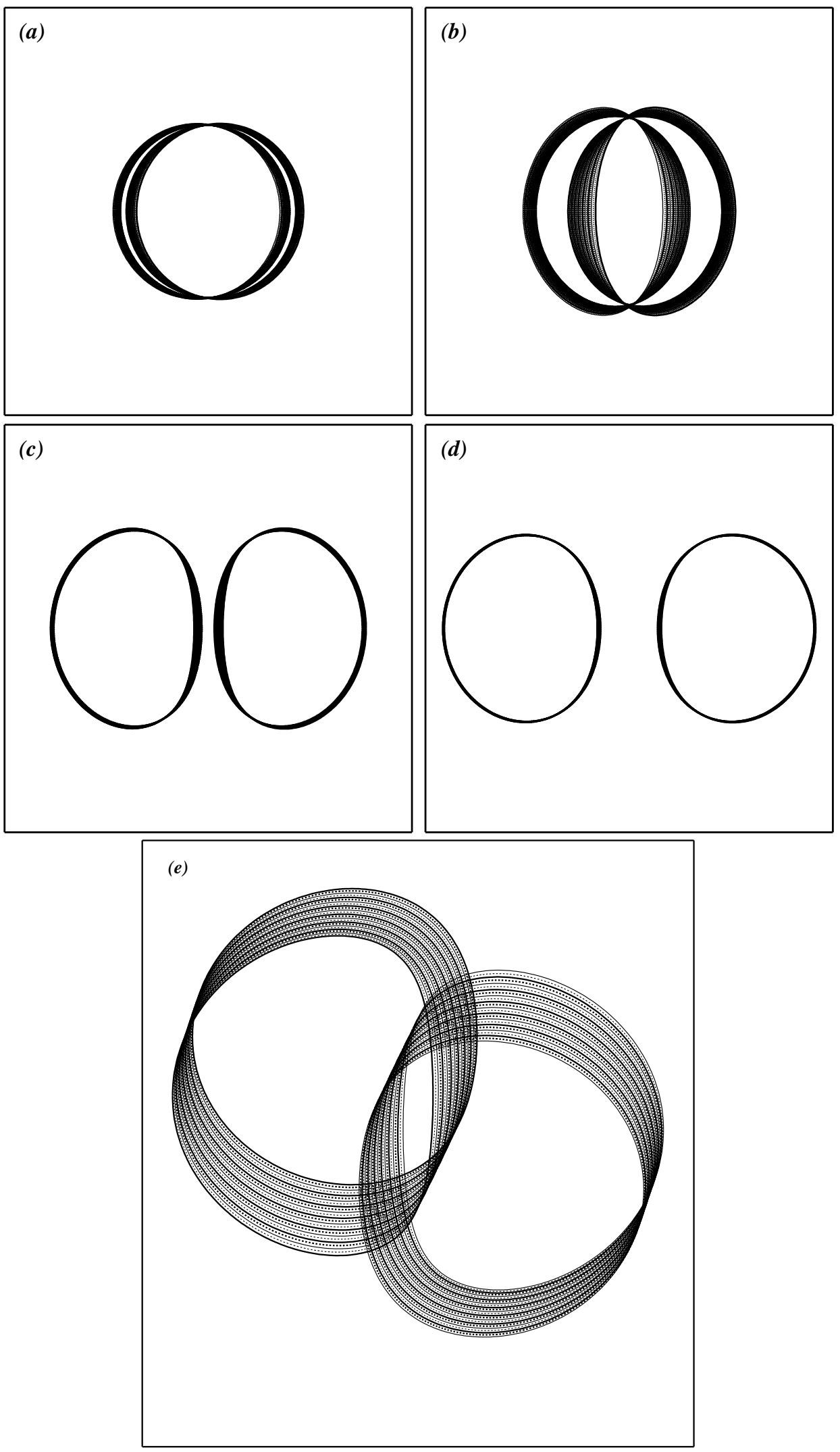

Figure 2. Top view of equilibria vortices for $r / h_{v}=4$, and $d / h_{v}=1$. Figures are (a) $\delta / r=2.23,(b) \delta / r=2.49,(c) \delta / r=3.69$ and $(d) \delta / r=4.37$. And at a view of $45^{\circ}$, (e) $\delta / r=2.83$ 

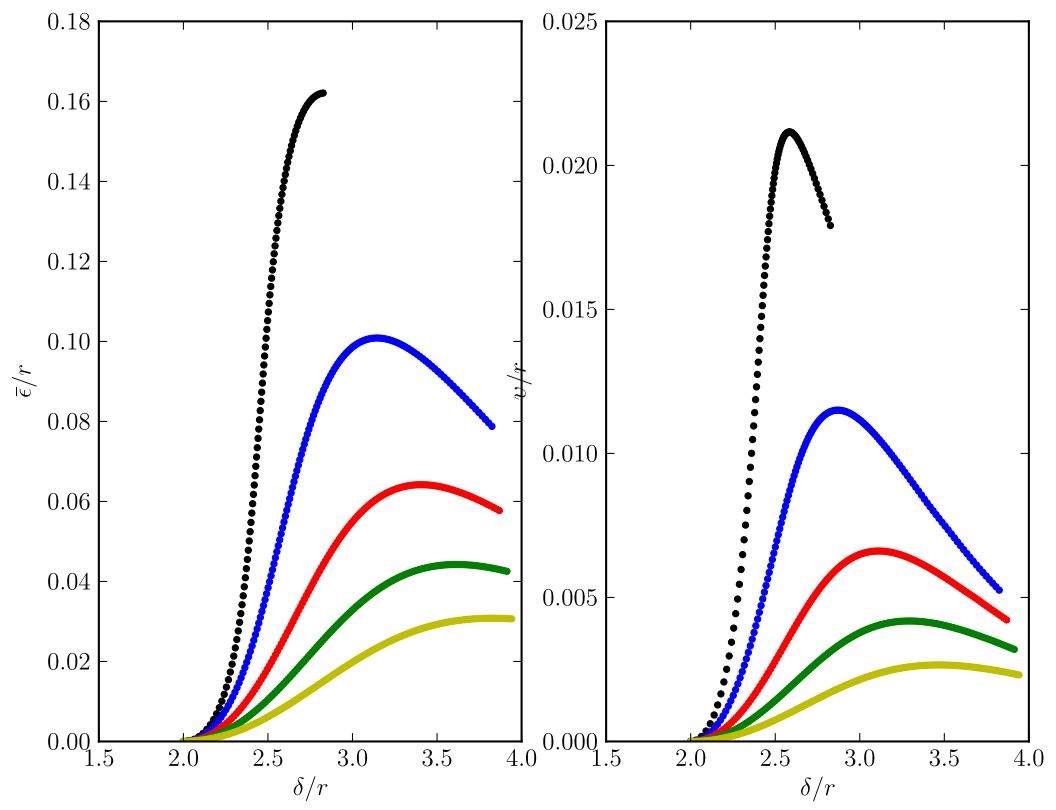

Figure 3. Relative deformation measures $\bar{\epsilon} / r$ and $v / r$ vs the gap $\delta / r$ for $r / h_{v}=4$ and $d / h_{v}=1$ (black), 2( blue), 3 (red), 4 (green), 5 (yellow).

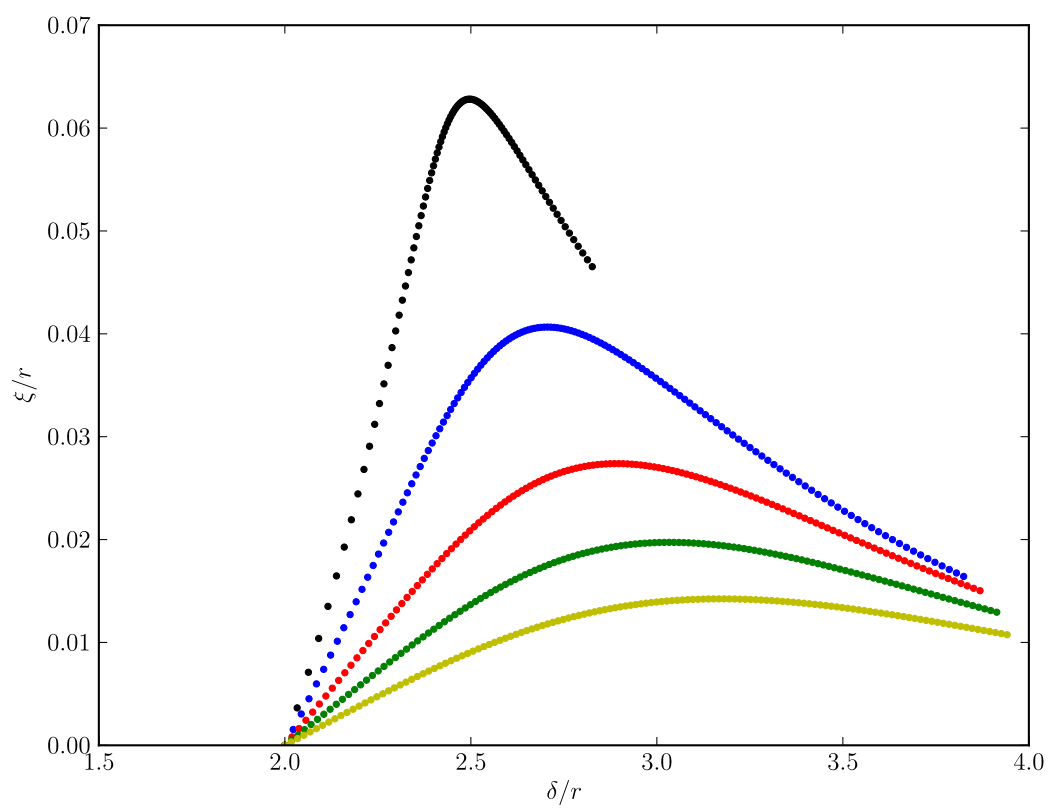

Figure 4. Relative centroid displacement variance $\xi / r$ vs the gap $\delta / r$ for $r / h_{v}=4$ and $d / h_{v}=1$ (black), 2( blue), 3 (red), 4 green, 5 (yellow). 
would not be available is each pole was mapped by a single layer. The deformation of the shape of poles of an equilibrium state is measured from its departure from a cylindrical shape with circular cross-section. Two measures are used for that purpose. The first one is related to the shape of the horizontal cross-sections of the vortices, the second one is is related to the vertical tilt of the structure. We start by determining the centroid location $\mathbf{X}_{c}(j)$ of each contour $j$ of the $n_{c}$ contours describing the equilibria $\left(n_{c}=2 n_{v}\right)$. We denote $\left(\mathbf{x}_{i}(j)\right)_{1 \leq i \leq n_{p}}$ the $n_{p}$ nodes describing the contour $j$ and $\rho_{i}(j)$ the local horizontal radius. Next, $\epsilon(j)$ measures the departure from a circular shape by calculating the standard deviation of the local horizontal radius $\rho_{i}(j)$ from the mean $\bar{r}(j)$ along the contours. $v$ measures a part of the vertical deformation by measuring the standard deviation of $\epsilon(j)$ across the contours. The second part of the vertical deformation is the tilt of the structure measured by the standard deviation $\xi$ of the contour centroid location $\mathbf{X}_{c}(j)$. The measures are summarised in the formulae (10-18) below.

$$
\mathbf{X}_{c}(j)=\frac{\iint_{\mathcal{A}_{j}} \mathbf{x} \mathrm{d} A}{\iint_{\mathcal{A}_{j}} \mathrm{~d} A},
$$

In practice, the surface integrals are transformed into contour integrals, 1.e.

$$
x_{c}=\frac{\oint_{C_{j}}(1 / 2) x^{2} d y-x y d x}{\oint_{C_{j}} x d y-y d x},
$$

where $C_{j}$ bounds $A_{j}$. Note that $y_{c}=0$ by construction and $z_{c}$ is fixed.

$$
\begin{aligned}
& \overline{\mathbf{X}}_{c}=\frac{1}{n_{c}} \sum_{j=1}^{n_{c}} \mathbf{X}_{c}(j), \\
& \rho_{i}(j)=\left|\mathbf{x}_{i}(j)-\mathbf{X}_{c}(j)\right|, \\
& \bar{r}(j)=\frac{1}{n_{p}} \sum_{i=1}^{n_{p}} \rho_{i}(j), \\
& \epsilon(j)=\frac{1}{\sqrt{n_{p}}} \sqrt{\sum_{i=1}^{n_{p}}\left(\rho_{i}(j)-\bar{r}(j)\right)^{2}}, \\
& \bar{\epsilon}=\frac{1}{n_{c}} \sum_{j=1}^{n_{c}} \epsilon(j), \\
& v=\frac{1}{\sqrt{n_{c}}} \sqrt{\sum_{j=1}^{n_{c}}(\epsilon(j)-\bar{\epsilon})^{2},} \\
& \xi=\frac{1}{\sqrt{n_{c}}} \sqrt{\sum_{j=1}^{n_{c}}\left(\mathbf{X}_{c}(j)-\mathbf{X}_{c}\right)^{2}} .
\end{aligned}
$$




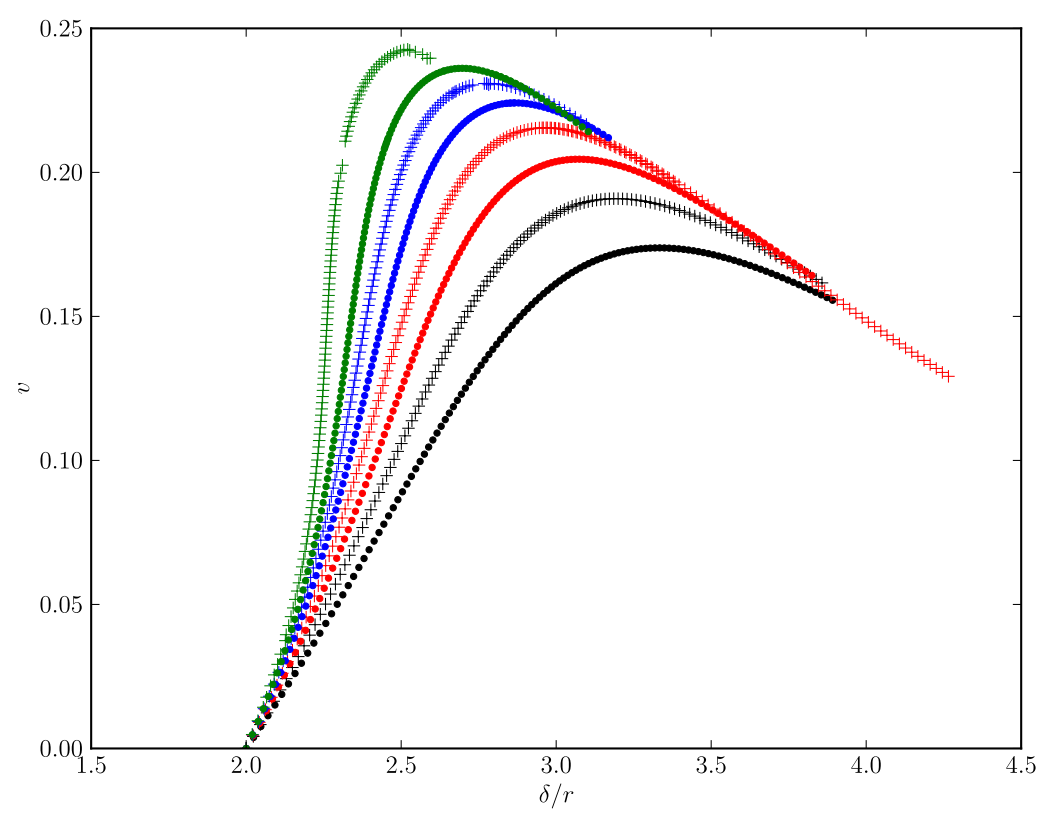

Figure 5. Translation velocity $v$ of the equilibria vs the gap $\delta / r$ for $r / h_{v}=3$ (black .), 3.5 (black +), 4.0 (red ·), $4.5($ red +$), 5$ (blue $\cdot), 5.5$ (blue +$), 6.0$ (green ·), 7.0 (green + ) and $d / h_{v}=2$. The velocity scales as the strength of the vortices divided by a distance squared and divided by $\pi$. Here the strength of each vortex is set to $q V_{v}=2 \pi^{2}$, so the velocity scales as $\pi /\left(2 r^{2}\right)$

Results for the horizontal shape of the vortices are presented in figure 3 for $r / h_{v}=4$. We see that the relative standard deviation of the radius reaches values of order $10^{-1}$ (with maxima, as a function of $\delta / r$, in the range $0.04-0.16$ ). This means that the standard deviation can be about $10 \%$ of the radius. More importantly, the vertical standard deviation of this value is again of about $10 \%$ of the mean. This implies that there is a significant vertical variation of the vortex horizontal shape, even is the structure itself is rather flat. Recall $r / h_{v}=4$ means that the vortex is typically 4 times wider than tall. The vortices are also tilted as shown in figure 4 with the standard deviation for the centroid reaching typically a few percents of the vortex radius. The deformations reach peaks at finite separation distances as anticipated.

Figure 5 shows the translation velocity $v$ of the equilibria vs the relative gap $\delta / r$ for a fixed vertical offset $d / h_{v}=2$ and various values of the aspect ratio $r / h_{v}$. To be able to compare the various cases, the scaling is set by imposing that the strength (volume integral of PV) is the same in all cases as well as the PV itself. The strength alone determines the far field while the PV determines the rotation rate of the poles. Indeed, the PV sets a time scale $T$. For example, the turnover period of a sphere of $\mathrm{PV} q$ is $T_{t}=6 \pi / q$, here $\mathrm{PV}$ is set to $2 \pi$. The total volume $V_{v}$ of each pole is set to $\pi$, i.e. $V_{v}=2 h_{v} r^{2} \pi=\pi$, which means $r^{2} h_{v}=1 / 2$. Therefore, by denoting the aspect ratio $\alpha=r / h_{v}$, the height scale $h_{v}$ associated with the problem is such $\alpha^{2} h_{v}^{3}=1 / 2$, $h_{v}=\sqrt[3]{0.5 \alpha^{-2}}$. 
To analyse the translation velocity, we first compared it to the one of equivalent singular point vortices. The translation velocity associated with singular hetons corresponding to charges of the same strength $\Gamma=q V_{v}=2 \pi^{2}$ and separated by $\ell$ in horizontal and $z$ in the vertical direction is

$$
v_{s}=\frac{\Gamma}{4 \pi} \frac{\ell}{\left(\ell^{2}+z^{2}\right)^{3 / 2}}=\frac{\pi}{2} \frac{\ell}{\left(\ell^{2}+z^{2}\right)^{3 / 2}}
$$

This estimate would be accurate if the poles of the hetons were non deformable spherical vortices. This is far from being the case in the present study as the vortices are flat structures (as we focus on the margin of stability of baroclinically unstable hetons), and their shape at equilibrium cannot be assumed in an ad hoc manner. The first determinant factor is the fact that the vortices are here flat structure (typically from three up to eight times wider than tall). This means that the PV is concentrated on fewer, larger vertical section compared to a same volume sphere. A first influence of the shape of a vortex on the velocity field it induces can be obtained representing vortices by ellipsoids, which is the next degree of refinement from a single charge at the centre. Even then, the external field is non-trivial and discussed in Dritschel, Reinaud and McKiver (2004) and Dristchel, Scott and Reinaud (2005).

A comparison between the full calculation and the translation velocity of equivalent charges located at the centre $x_{c}$ of each vortex is provided in figure 6 for $d / h_{v}=1$ and various $r / h_{v}$.

We also include a stable configuration in which the family of equilibria for $r / h_{v}=1$ was additionally computed. Note that this family is stable to baroclinic modes. In this case, the vortices are likely to be well modelled by singularities located at the poles centres. The figure 6 indeed shows that for compact vortices the approximation is accurate. But as soon as we depart from near unit height-to-width aspect ratio vortices, the point vortex approximation fails to capture the translation velocity of the structure at close range. Convergence between the two models is recovered at long range, but this is not the purpose of the current study.

We mainly focus on the margin of stability of hetons for baroclinic modes, i.e. hetons consisting of wide, close poles. To this purpose we first need to determine equilibria. The procedure has a numerical cost which is proportional to the square of the number of points used to discretize them, hence determining equilibria is a numerically expensive problem. There is little interest in calculating stable states.

At closer range, which is our focus, the actual shape of the equilibria is key to measure the hetons translation velocity. Nevertheless, qualitatively, the point vortex approximation still shed some light on global trends for the phenomena. Indeed, as expected from a point vortex calculation the velocity is non-monotonic as a function of the horizontal separation. It first increases from zero as the vortices are pushed further apart then reaches a maximum before slowly decaying. The figure shows indeed a strong influence of the aspect ratio of the vortices on the translation velocity. It is shown that flatter vortices may produce higher translation velocity for the same overall volume of PV. We attribute this to the fact that vortices overlap more as we separate them in the 

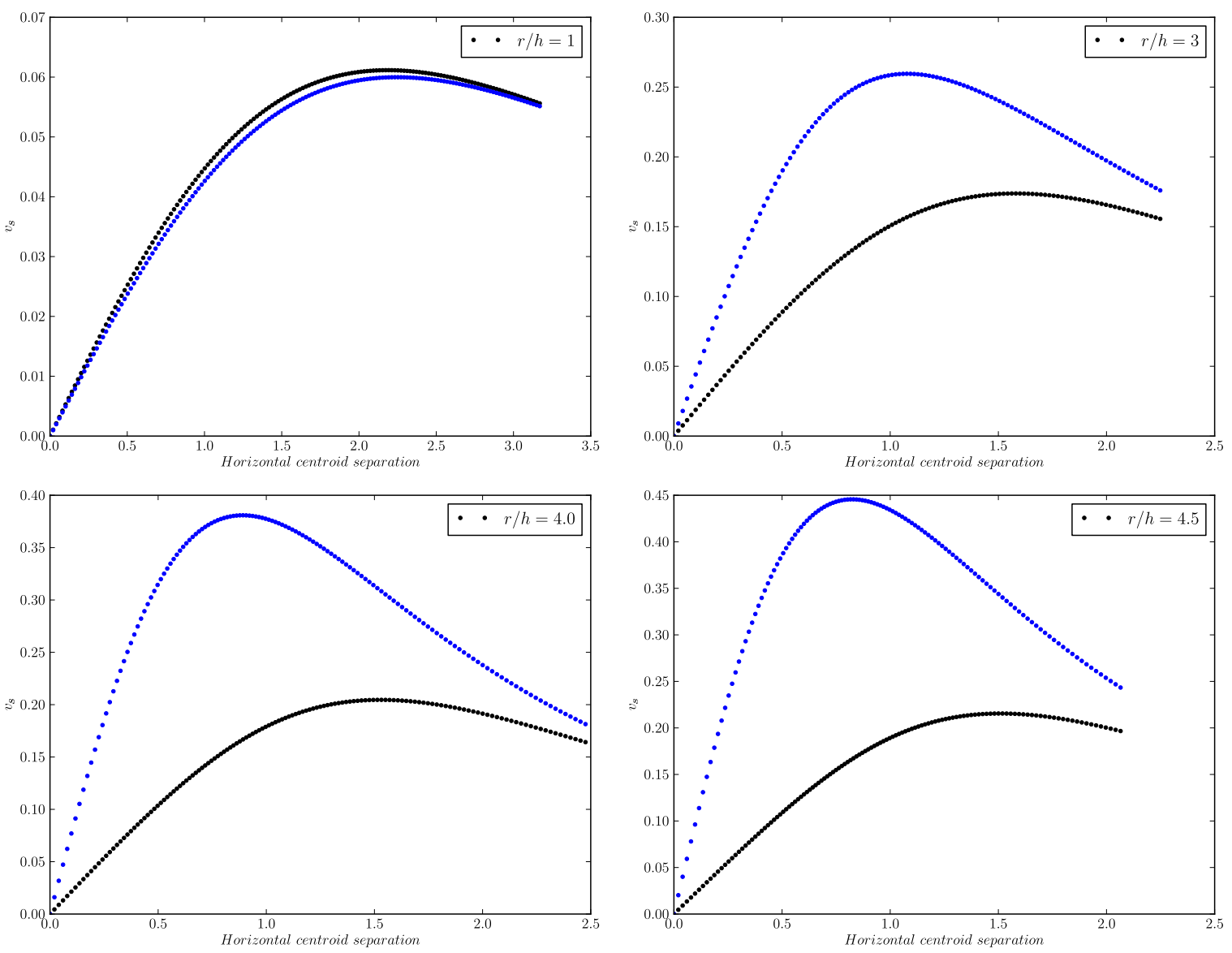

Figure 6. Comparison of the translation velocity of the flat equilibrium hetons and the translation velocity of singularities at close range in the case $r / h_{v}=1,3,4,4.5$. Equilibria (black), point vortex (blue).

horizontal.

Figure 7 shows the translation velocity $v_{s}$ vs $\delta / r$ for $r / h_{v}=7$ and various values of vertical offset $d / h_{v}$. Here, with $r / h_{v}$ fixed, the trend is easier to understand from the crude point vortex model as the horizontal extend of the vortex is comparable in all cases. For any given horizontal distance, the velocity decreases with the vertical distance (hence the overall distance) as the velocity decays as the inverse squared of the three dimensional distance. On the other hand, the maximum velocity in the point vortex approximation is obtained at $\ell=z$ (from a point vortex calculation). Therefore, one expects the maximum velocity of the equilibria to be reached at larger gaps, as $d / h_{v}$ is increased.

We next turn our attention to the stability properties of the equilibria. The structure of the modes analysed is briefly described in the appendix. The modes allow both the deformation of the contours as well as the tilt of the vortices. Figure 8 presents the complex eigenvalues $\sigma$ of the normal modes vs the gap $\delta$ for $r / h_{v}=4$ and $d / h_{v}=1$. The modes are proportional to $e^{\sigma t}$. Therefore, the real part $\sigma_{r}$ of $\sigma$ corresponds to 


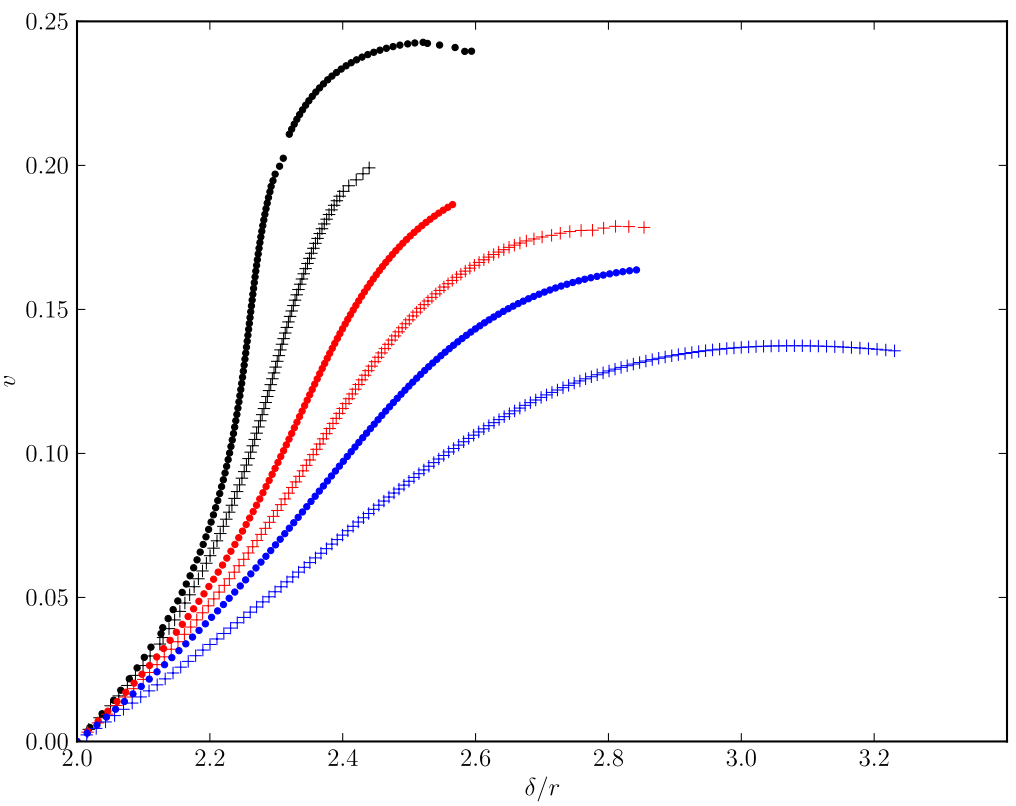

Figure 7. Translation velocity $v$ of the equilibria vs the gap $\delta / r$ for $r / h=3.5$ and $d / h_{v}=2$ (black ·), 2.5 (black + ), 3 (red $\left.\cdot\right), 3.5($ red +$), 4$ (blue $\left.\cdot\right), 5$ (blue + ). The velocity scales as the strength of the vortices divided by a distance squared and divided by $\pi$. Here the strength of each vortex is set to $q V_{v}=2 \pi^{2}$, so the velocity scales as $\pi /\left(2 r^{2}\right)$

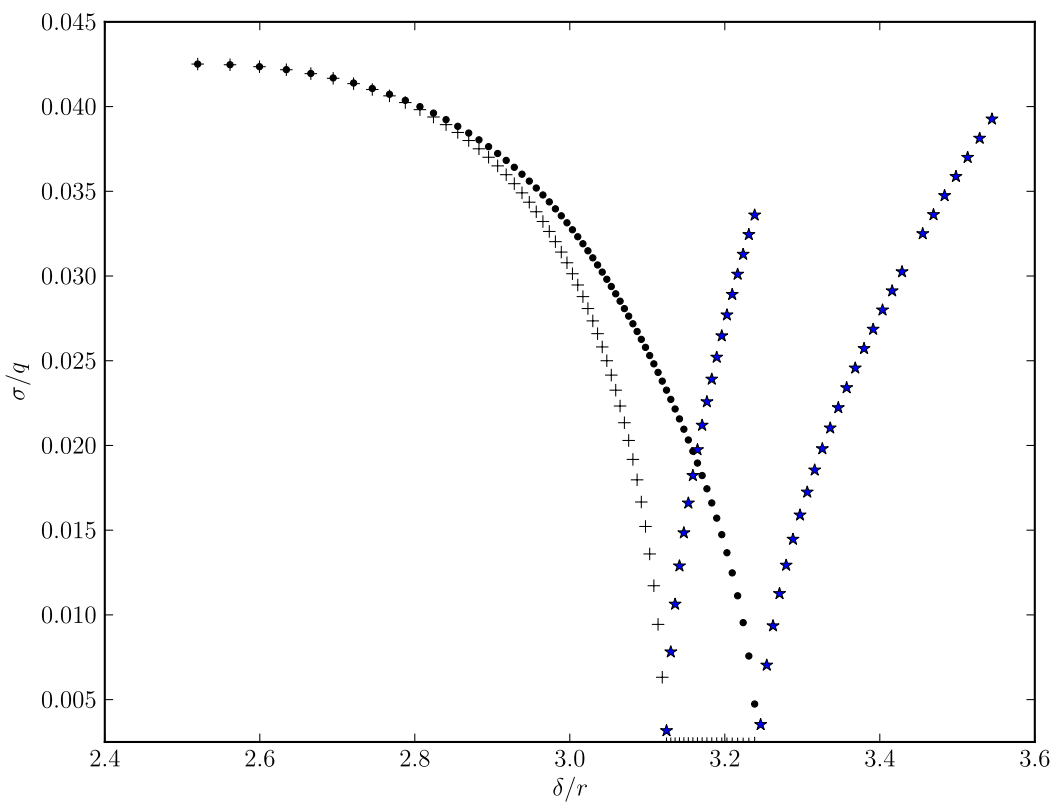

Figure 8. Eigenvalues $\sigma / q$ corresponding to the two most unstable modes for $r / h_{v}=4$ and $d / h_{v}=1$, where $q=2 \pi$ is the PV. The growth rate of the most unstable mode $(\cdot)$, second unstable mode $(+) .(\star)$ indicate the frequency of the modes before the margin of stability. 


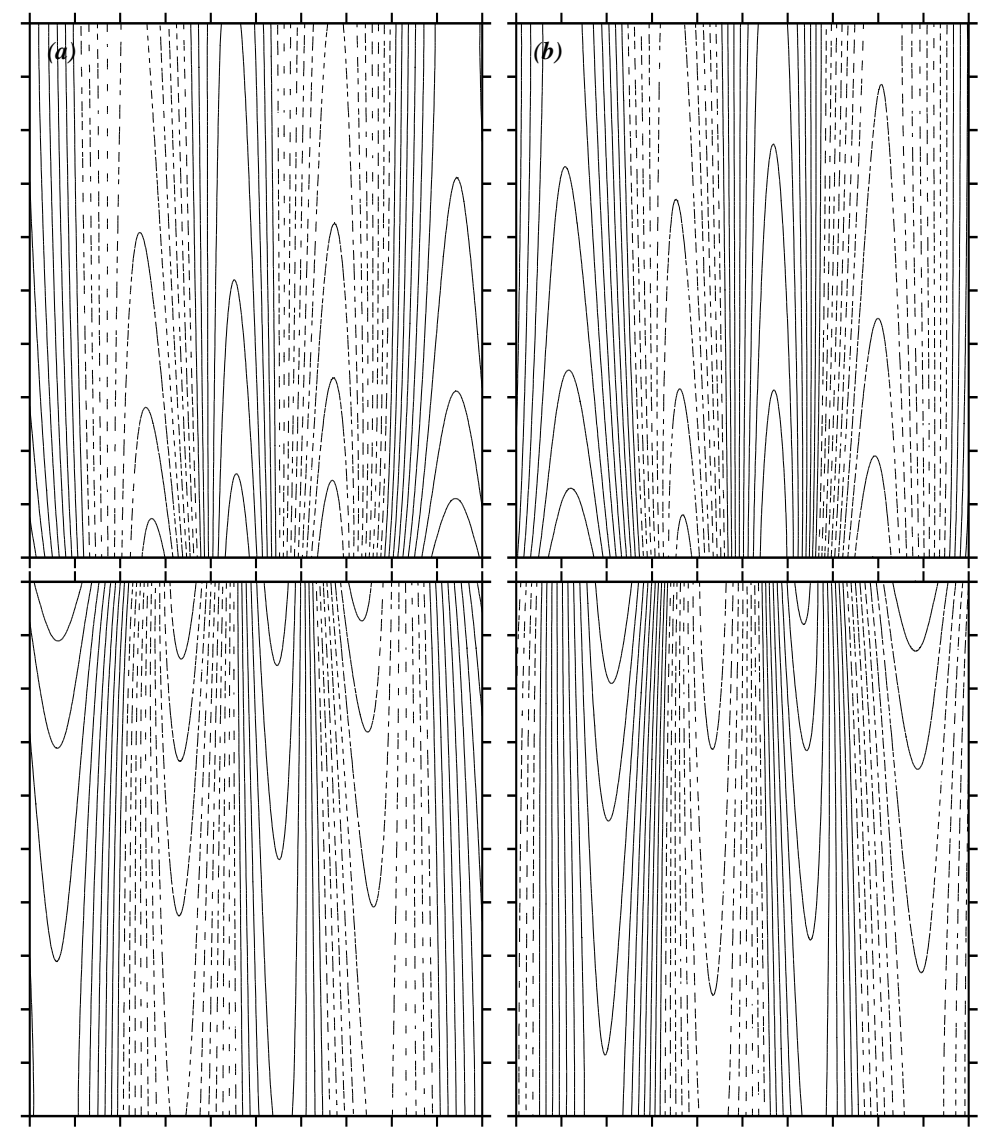

Figure 9. Spatial structure (contour plot of the imaginary part of the eigenvectors) of the two unstable modes for $r / h_{v}=4, \delta / r=2.44$ and $d / h_{v}=1$. (a) the most unstable mode and $(b)$ the second most unstable mode. In each case the top figure represent the top vortex while the bottom figure represents the bottom vortex.

growth rate of the mode while the imaginary part $\sigma_{i}$ is its frequency. In this case (and this is generic for the modes along this family of equilibria) the eigenvalue $\sigma$ is such that $\sigma^{2} \in \mathbb{R}$, i.e. $\sigma$ is real or pure imaginary. From neutrally stable equilibria $\left(\sigma=i \sigma_{i}\right)$ the frequency of a mode decreases rapidly as the gap $\delta$ is reduced and eventually collapses to zero at $\delta=\delta_{m}$. Then the mode becomes unstable for smaller gaps (the eigenvalue becomes real with $\sigma=\sigma_{r}$ ). Figure 8 shows that the growth rate of the modes increases as the gap (and the overall distance) between the two vortices is decreased. We should note that the modes in fact come in pairs. If $\sigma$ is an eigenvalue, so is $-\sigma$. Each unstable mode is therefore coupled with a stable one. The figure shows the emergence of two unstable modes (we do not represent the associated stable modes as their eigenvalues are symmetric with respect to the horizontal axis). However, we see that the two modes have growth rates which are converging together as the gap is reduced.

The spatial structure of the modes is presented in figure 9 for $\delta / r=2.44$. The figure shows contour plots of the eigenvectors, i.e. lines of iso-deformation. The solid lines corresponds to negative values (i.e. deformations toward in the interior of the structure) while the dashed lines corresponds to deformations outward. The vertical 
axes are the heights of the vortices. The top figures correspond to the top vortex while the bottom figures correspond to the bottom one. The horizontal axis corresponds to a labelling of the nodes mapping the contours. It corresponds exactly to the polar angle for the axisymmetric case as the nodes are initially evenly distributed between 0 and $2 \pi$. Figure $9(a)$ shows the spatial structure of to the most unstable mode while $9(b)$ shows the second most unstable mode. We note that in both case, the deformation presents two positive ranges and two negative ranges in alternate order, corresponding in the axisymmetric basic state $(\Delta x=0)$ to the azimuthal mode $m=2$. Here it is not strictly speaking $m=2$ because the basic state is no longer axisymmetric, but the modes along the branch stem from $m=2$. We also recover the angular shift in the deformation of the two vortices. This is generic of the baroclinic instability, as pointed out in Reinaud \& Carton (2009). We first recover that the deformation varies within the height of the vortices, parts of a given vortex closest to the other one being more deformed, due to the increased mutual influence. Comparing the spatial structure of the two unstable modes we obverse that, qualitatively, they are similar apart for the phase between them. However, there is a slight difference in the vertical structure of the two modes. By calculating the norm of the deformation associated with the modes along each layer, we observe that the most unstable mode corresponds to a deformation which varies more in height inside the vortices. We could say that it is more 'baroclinic'. To measure the difference between the two modes, we measure the square of the norm of the eigenvector for each contour of the vortex 1 and compare it to the overall mean to obtain the variance. The vertical variance is 0.738 for the most unstable mode and 0.559 for the second most unstable mode. Here the difference is small but enough to explain the asymmetry between the two modes, and is observed in all configurations where the two modes differ. Recall that the two modes have the same growth rate when the two (cylindrical) vortices are vertically aligned by symmetry. Note that this distinction would be impossible if the poles were modelled by a single layer.

The nonlinear evolution of the flow with $r / h_{v}=4, d / h_{v}=1$ and $\delta / r=2.44$ is illustrated in figure 10. The heton remains close to its equilibria shape up to $t \simeq 40$, then the deformation becomes noticeable. The two poles deform in a shape consistent with the $m=2$ mode and eventually the central part of the elongated poles shrinks to form dumbbell-like vortices. The PV bridge connecting the new poles of the dumbbells eventually breaks and the heton breaks into two separate hetons which start to translate apart. In the later evolution (not shown) the hetons briefly re-interact before translating away from one another.

We next summarise results over a larger parameter space. The aspect ratios investigated are $r / h_{v}=3,3.5,4,4.5,5,5.5,6,6.5,7,7.5$ and 8 . The vertical offsets are $d / h_{v}=1,2,3,4$, and 5 . Two additional vertical offsets $(2.5 \& 3.5)$ have been used for $r / h_{v}=7$ for illustration purposes. Unfortunately, in practise not all branches could be obtained in full. We focus on the two most unstable (dominant) modes.

Figure 11 shows the growth rates of the two most unstable modes vs $\delta$ for $r / h_{v}=7$ and $d / h_{v}=2,2.5,3,3.5$, and 4 . The effect of increasing the vertical offset is to weaken 

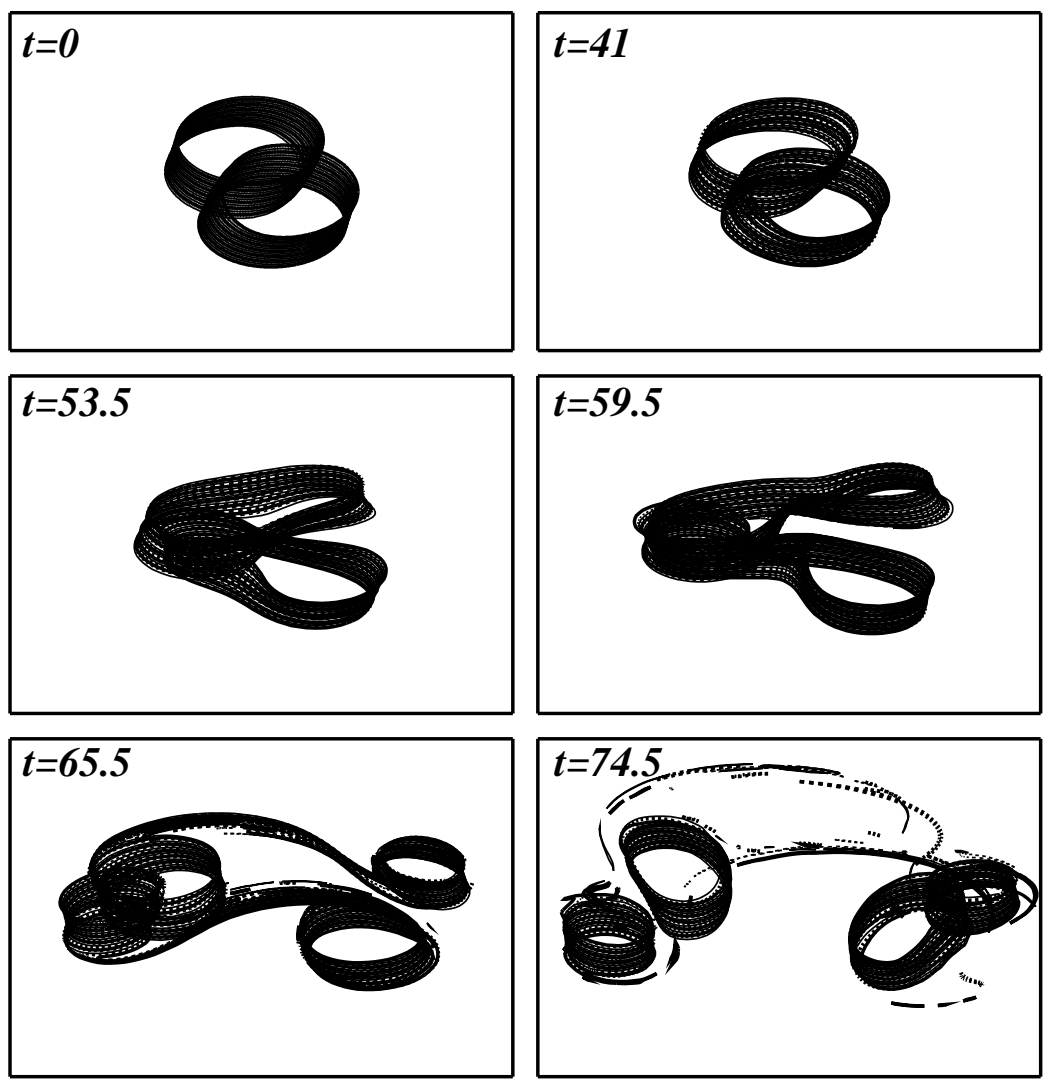

Figure 10. Time evolution of the unstable equilibria for $r / h_{v}=4, \delta / r=2.44$ and $d / h_{v}=1$. Each image is centred at the global point coordinates average.

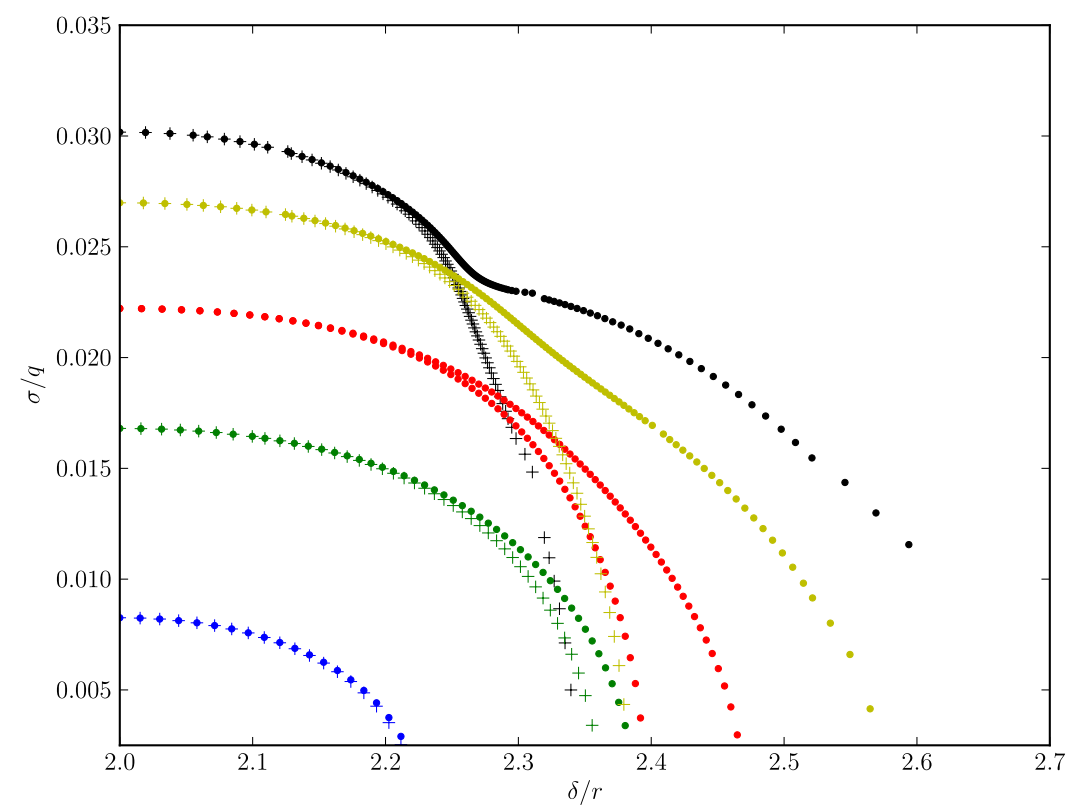

Figure 11. Growth rates $\sigma$ of the two unstable modes vs the gap $\delta$ for $r / h_{v}=7$ and $d / h_{v}=2$ (black), 2.5 (yellow), 3 (red), 3.5 (green), 4 (blue). 

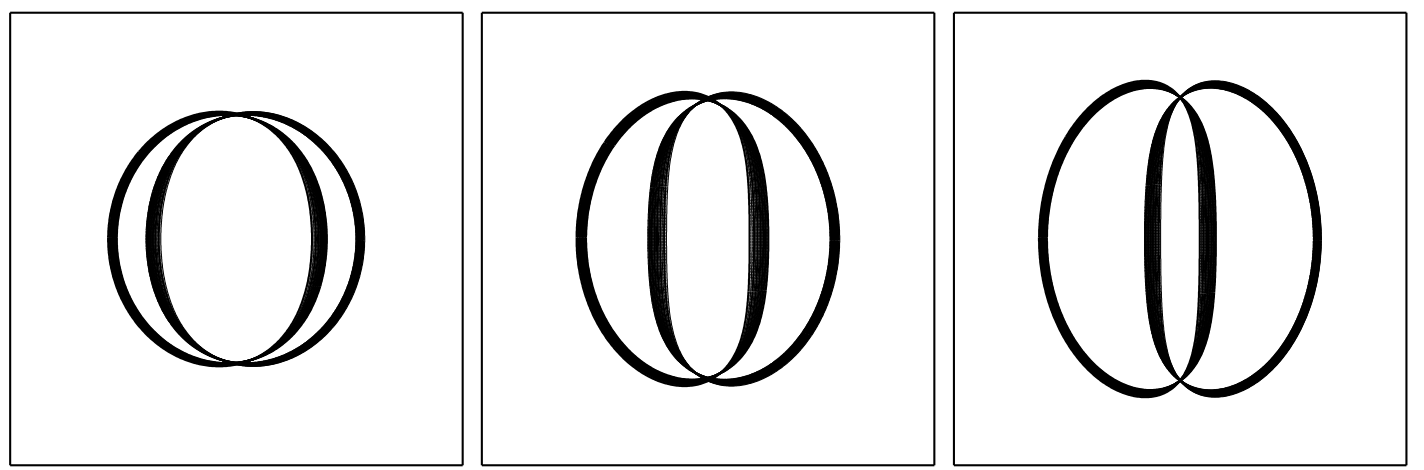

Figure 12. Top view of the equilibria for $r / h_{v}=7$, and $d / h_{v}=2$ and $(a) \delta / r=2.24$ (b) $\delta / r=2.30$ and $(c) \delta / r=2.44$.

the instability as the growth rates are smaller and the critical horizontal gap $\delta_{m}$ beyond which the heton stable becomes smaller. This is arguably a consequence of the increasing overall distance between the two vortices. More interestingly, the difference between the two most unstable modes increases as the gap is increased. Recall that the two modes are equivalent dynamically when $\Delta x=0$, which is $\delta=2 r$ where the outermost gap is the diameter of the cylindrical configuration. This shown that one of the modes becomes predominant when adding vertical variation such as in the shape of the vortices. This is consistent with the observation that the modes defer from their vertical structure of the modes as pointed out in a previous paragraph. In the case of $r / h_{v}=7$, the curve describing the evolution of the growth rate vs the gap even changes concavity, most noticeably for $d / h_{v}=2$. Nonetheless, the variation of all data describing the branch vary smoothly indicating that this is not a bifurcation. The shape of three equilibria along the branch is presented in figure 12. The spatial structure of the most unstable mode remains qualitatively the same along branch. This is illustrated in figure 13. All indicates that they indeed belong to the same family of equilibria.

Figure 14 shows the influence of the aspect ratio $r / h_{v}$ on the instability for a fixed $d / h_{v}=2$. The figure first confirms the higher sensitivity to instability of wider vortices, for at least the most unstable mode. We see that not only for a given gap $\delta$ the growth rate of the instability (in the range sought) is higher the wider the vortex is, but the extend of the range in $\delta$ sensitive to the instability increases (i.e. $\delta_{m}$, the gap at the margin of stability, increases). Due to the increased asymmetry between the two most unstable modes, the second mode is stabilised for shorter gap. This gap may be smaller than the marginal gap for the most unstable mode of narrower vortices. For $r / h_{v}=7$ the second mode becomes stable for $\delta / r>2.35$ while the most unstable mode remain unstable for $\delta / r<\delta_{m} / r=2.65 . \quad \delta=3.56$ is smaller than the margin of stability corresponding to the most unstable mode for $r / h=3$ and 3.25 .

Results are summarised in table 1 . The information provided in the table is as follows. The table shows the overall margin of stability (gap $\delta_{m}$ ) for various values of the aspect ratio $r / h$ and vertical offset $\Delta z / h$ when available. We indicate by a symbol 


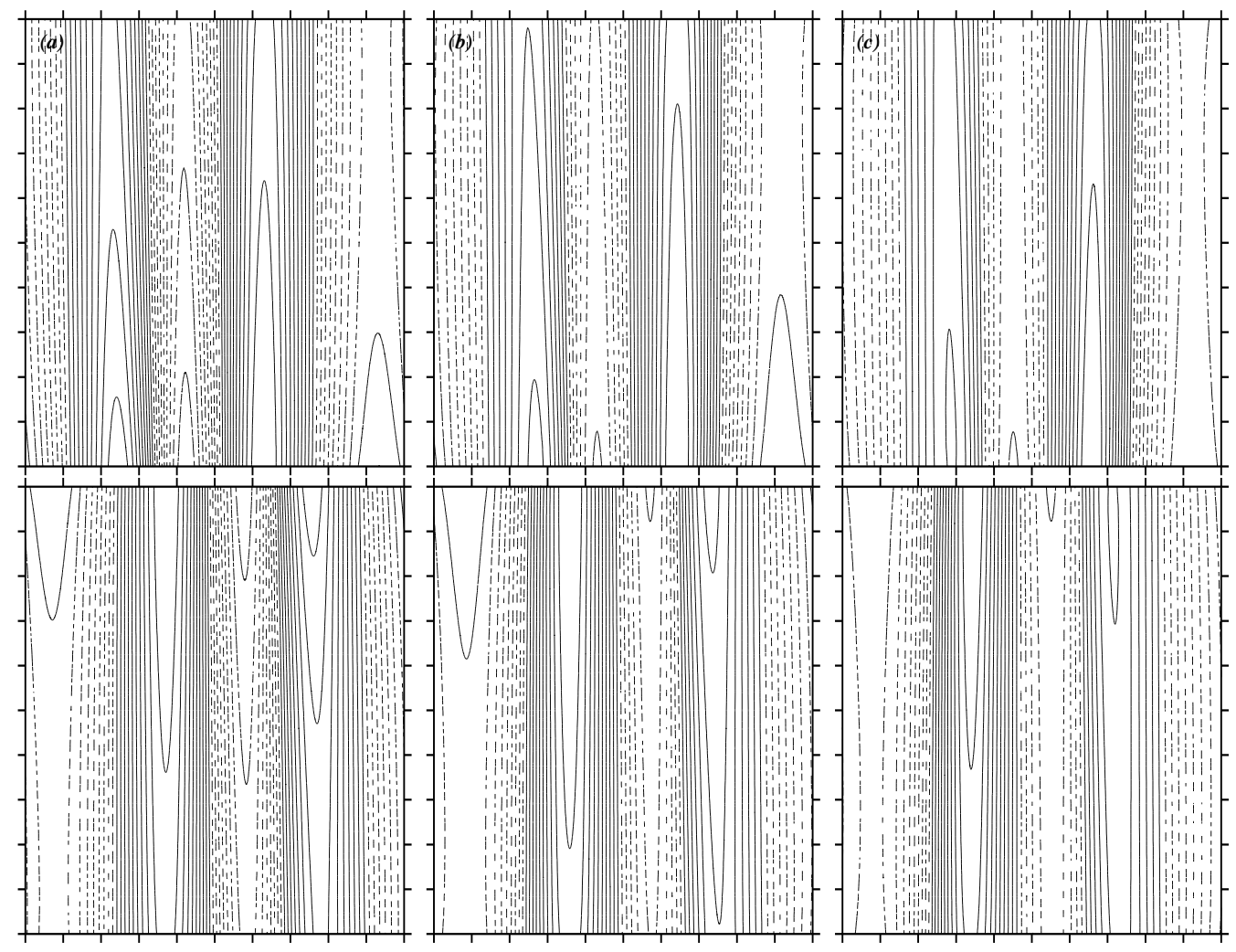

Figure 13. Spatial structure of the most unstable modes for $r / h_{v}=7$, and $d / h_{v}=2$ and $(a) \delta / r=2.24(b) \delta / r=2.30$ and $(c) \delta / r=2.44$. In each case the top figure represents the top vortex while the bottom figure represents the bottom vortex.

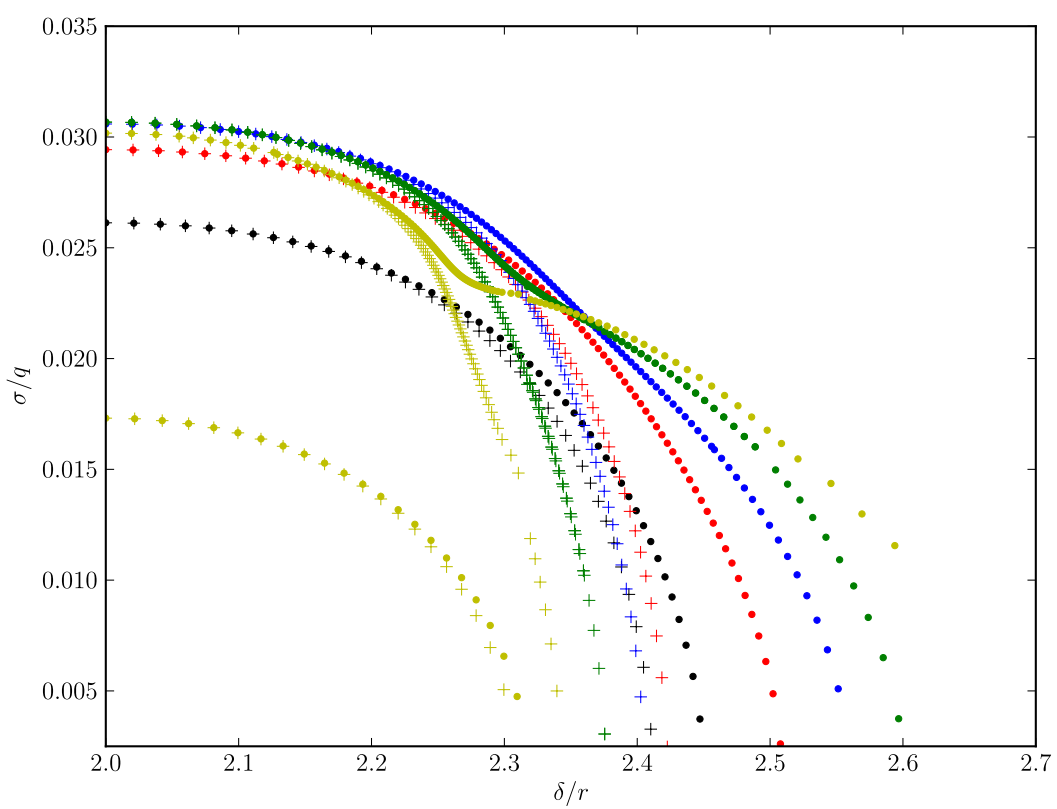

Figure 14. Growth rates $\sigma / q$ of the two unstable modes vs the gap $\delta / r$ for $r / h_{v}=4.5$ (yellow - upper curves), 5 (green), 5.5 (blue), 6 (red), 6.5 (black), 7 (yellow - lower curves) and $d / h_{v}=1$. 


\begin{tabular}{c|ccccccccccc}
\hline$d / h_{v}-r / h_{v}$ & 3 & 3.5 & 4 & 4.5 & 5 & 5.5 & 6 & 6.5 & 7 & 7.5 & 8 \\
\hline 1 & 2.26 & 2.51 & 2.57 & 2.62 & 2.75 & $\times$ & $\times$ & $\times$ & $\times$ & $\times$ & $\times$ \\
\hline 2 & $\circ$ & $\circ$ & $\circ$ & 2.31 & 2.45 & 2.51 & 2.56 & 2.60 & 2.65 & $\times$ & $\times$ \\
\hline 3 & $\circ$ & $\circ$ & $\circ$ & $\circ$ & $\circ$ & $\circ$ & 2.32 & 2.42 & 2.46 & 2.52 & $\times$ \\
\hline 4 & $\circ$ & $\circ$ & $\circ$ & $\circ$ & $\circ$ & $\circ$ & $\circ$ & $\circ$ & 2.18 & 2.36 & 2.43 \\
\hline 5 & $\circ$ & $\circ$ & $\circ$ & $\circ$ & $\circ$ & $\circ$ & $\circ$ & $\circ$ & $\circ$ & $\circ$ & $\circ$ \\
\hline
\end{tabular}

Table 1. Stability diagram: $\circ($ stable for all $\delta)$, figure $\left(\delta_{m} / r\right), \times($ unstable for at least small $\delta$ but $\delta_{m}$ not reached numerically.)

(o) cases where $\delta_{m}$ does not exist (or can be seen as $\infty$ ), i.e. the heton is stable for all $\delta$. As previously mentioned, some branches could not be completed and we were not able to reach $\delta_{m}$ in practice. However, for these branches we can calculate the stability for the first few steady states (corresponding to small $\delta$ ), and indeed always for the first state corresponding to the original cylindrical state with $\Delta x=0$. If, at least the first state is unstable, indicating the existence of a range of $\delta$ for which the configuration is unstable, we indicate it by another symbol $(x)$. These figures illustrate quantitatively the trends previously discussed.

The upshot of the study of these families of equilibria is that hetons are stabilised rather rapidly when slightly offset in the horizontal, even if, as expected wide vortices remain the most unstable. The second important result is the non-negligible influence of the vertical structure of the equilibria and of the vertical structure of the instability modes. Modes with a similar azimuthal structure have different properties due to their different vertical structure.

\subsection{Second families of symmetric equilibria}

In the previous section, we have indicated that not all the branches of solutions could be obtained. One of the reasons is the existence of other branches of solutions with the same symmetries in the vicinity of the branch sought. In practice, the iterative method used to obtain the equilibria may 'jump' onto the alternative branch. Because the 'new' branch shares the same symmetries as the targeted one, it is impractical to find simple ways to prevent the code from accessing it (e.g. by imposing symmetries or attempting to break symmetries). For the same reasons it is impractical to study the full new branches nor it is the purpose of this contribution. However, we offer a few examples of the results obtained, with no attempt to be comprehensive.

This first secondary branches observed are for the smallest vertical offset investigated $d / h_{v}=2$, and $r / h_{v}>5$. In these cases, for the original branch of equilibria, not only the two modes $m=2$ are unstable, but the two next modes $m=3$ are also unstable, although their growth rate is smaller. For example, for $r / h_{v}>5.5, d / h_{v}=1$ and $\Delta x=0$ (case included in Reinaud \& Carton (2009)), we have $\sigma(m=2)=0.0406 q$ while $\sigma(m=3)=0.0365 q$, where $q=2 \pi$ is the PV. For 


$$
\begin{array}{ccc}
\text { mode } & r / h_{v}=5.5 & r / h_{v}=6.0 \\
1^{\text {st }} & 0.0291 q & (0.0219+i 0.0160) q \\
2^{\text {nd }} & 0.0245 q & (0.0219-i 0.0160) q \\
3^{\text {th }} & 0.0113 q & (0.0163+i 0.0117) q \\
4^{\text {th }} & 0.0091 q & (0.0163-i 0.0117) q
\end{array}
$$

Table 2. Growth rates of the modes of instability for the two cases along the second branch shown in figure 15. The frequency is no longer necessarily zero. In that case, the eigenvalues have to be complex conjugates of one another. However, the spatial structure of the two conjugate modes is different.

$r / h_{v}=6$ we have $\sigma(m=2)=0.0427 q$ and $\sigma(m=3)=0.0386 q$. With the existence of this new instability, another different branch of equilibria emerges, co-existing with the original. This new branch has different stability properties, and shows a brutal change in the values of the translation velocity for example. By running the same computation at different resolutions, we were able to change the location where the method jumps from one branch to one another and hence, at least for a few states, have similar states on both branches. We briefly discuss these branches on two examples.

Firstly, the shape of the alternative equilibria includes a $\mathrm{m}=3$-like deformation as illustrated in figure 15 for $r / h_{v}=5.5$ (as well as $r / h_{v}=6$ ). We include in the figure the equivalent cases for the original branch. The equilibria shown on the second branch is the first one obtained after the jump compared to the 'same' case obtained when remaining on the original branch. Both the shape of the vortices along the second branch and their unstable modes have been checked vs various numerical resolutions. We see in particular that the frequency of the unstable modes are not necessarily zero. However in that case the modes are a pair of complex conjugates which appears to be a mathematical constraint of the formulation. We conjecture that these modes with non-zero frequencies may stem from another bifurcation along the branch of solutions from a original mode with zero frequency.

This branch clearly does not stem from two vertically aligned cylindrical vortices. Following the branch as the gap is increased shows a reduction of the $m=3$-type deformation (not shown). We conjecture that the vortices would tend back to vortices with circular horizontal cross-sections as $\delta \longrightarrow \infty$. In other words, the two branches may in fact converge together at infinity. We also conjecure that these vortices are in fact stemming from a fully-developed ' $m=3$ type shape'. This cannot be asserted as the complete branch connected all states along the branch cannot be filled. However the shapes of a few examples of equilibria along this potential branch give a convincing hint that it is the case. These equilibria are showed in figure 16. Unfortunately we do not know of any usable single parameter monotonically varying along the branch to follow it in a systematic way.

For illustration purposes we perform a nonlinear simulation of the last equilibria presented in figure 17. The state remains close to equilibria for a long time before 

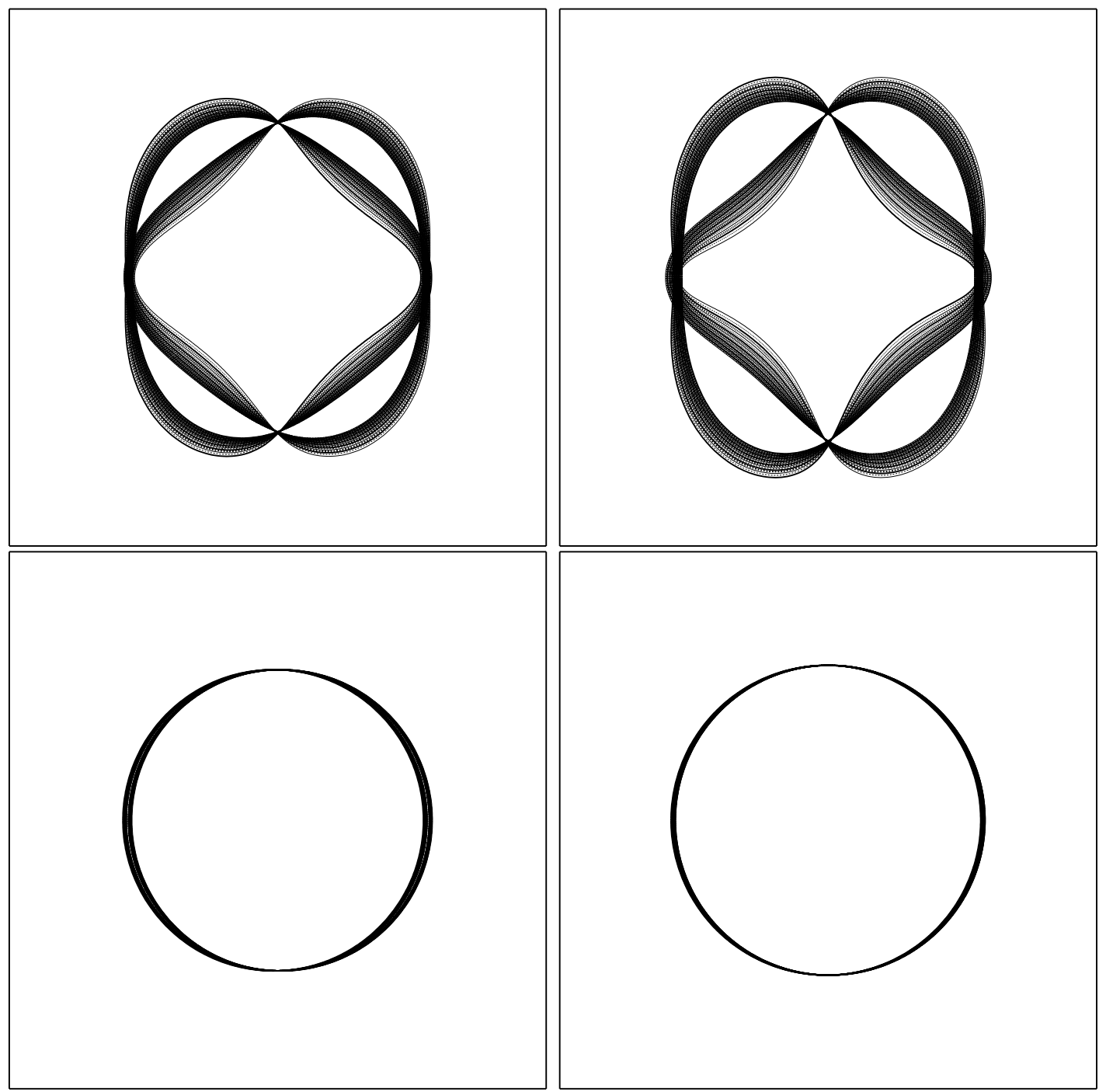

Figure 15. Top view of equilibria vortices (top: second family, bottom: first family) for $d / h_{v}=1$ and $r / h_{v}=5.5$ (left) or $r / h_{v}=6$ (right).

undergoing a rapid and destructive evolution where the original heton breaks into three secondary hetons which translate away from each other.

\subsection{Mode 2 and other symmetries}

As a final illustration of the complexity and richness of the problem we present a last result. In all the previous cases we had enforced a central symmetry onto the equilibria. It appears that both the original branch stemming from the vertically aligned cylindrical poles and the $m=3$ branch were compatible with this symmetry. But if the second branch is linked to the emergence of the mode $m=3$, a similar branch associated with the mode $m=2$ could exist. However, in the case, due to the phase in the deformation of the poles, The equilibria would not be compatible with the imposed symmetry. Therefore our investigation would have not be able to access it. Allowing for 

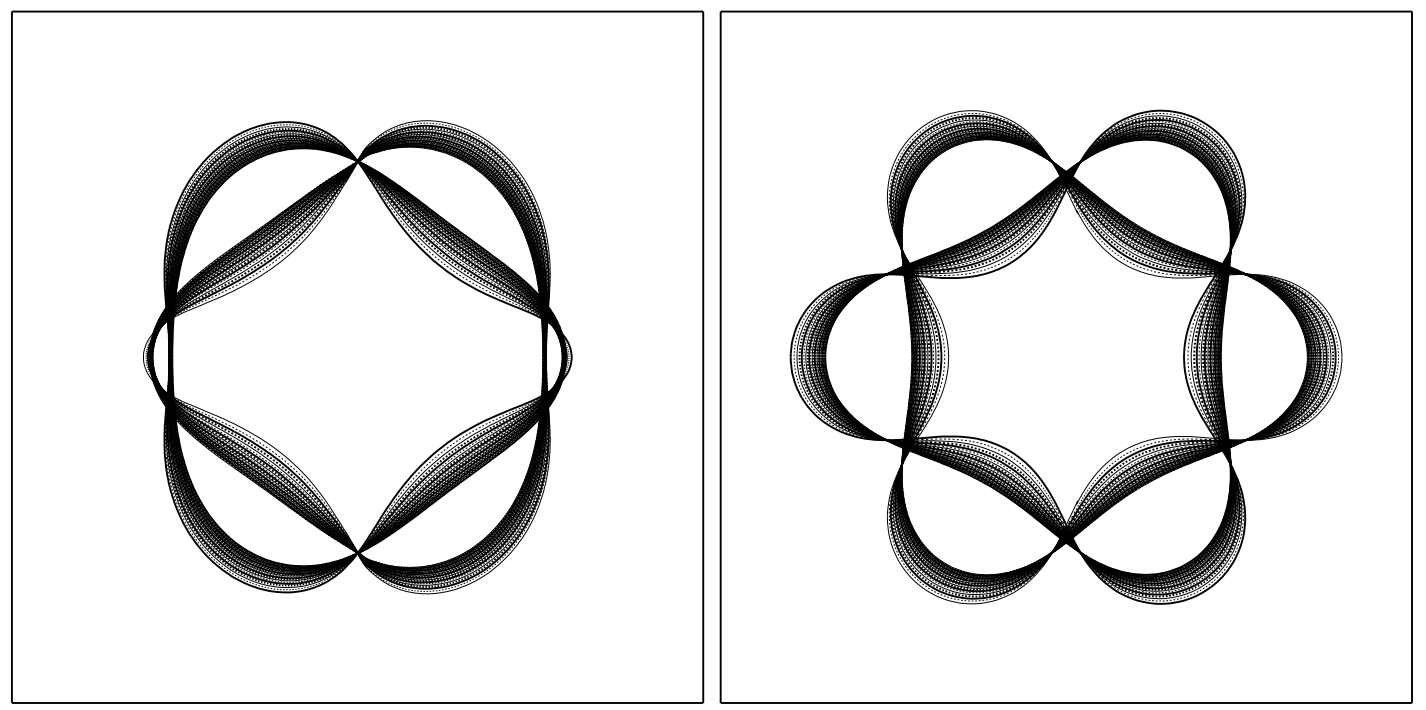

Figure 16. Example of equilibria shape for $r / h_{v}=5.5$ and $d / h_{v}=1$ along the second branch of equilibria.

non-symmetric shapes (apart from the natural symmetry with respect to the $x$-axis,) we were able to observe a mode 2-like equilibrium shape presented in figure 18 along the branch for $r / h_{v}=3.5$ and $d / h_{v}=1$. In this case mode 3 is not unstable but mode 2 is.

A linear stability analysis indicates that the heton of the secondary branch is unstable. A nonlinear simulation (not included in the paper) shows a rapid near realignment of the horizontal axis of the poles followed by a rapid breaking of the structure into multiple hetons. This isolated result hints the existence of many other branches of equilibria in the problem.

\section{Conclusion}

This paper studied hetons at equilibrium stemming from the case of a cylindrical vertically aligned heton. Branches of solutions, spanned by the horizontal offset, have been investigated for various aspect ratios and various vertical offsets between the poles of the heton. The main result of the study is that the instabilities observed in the aligned case disappear rather rapidly as the poles are offset in the horizontal, leading to stably translating structures. It therefore confirms that hetons are indeed likely to be very efficient means to transport heat and other properties carried by the vortices. This is also consistent with the results in two-layer models, for example Gryanik, Sokolovskiy and Verron (2006) where there is no separation in the vertical between the poles. And a similar conclusion may be found in Gryanik et al. (2000). We also recover that wide vortices are the most unstable. This study made use a continuous stratification approach, where many baroclinic modes are allowed within the vortices themselves. This proved to be very important in the dynamics. Firstly the shape of the equilibria 

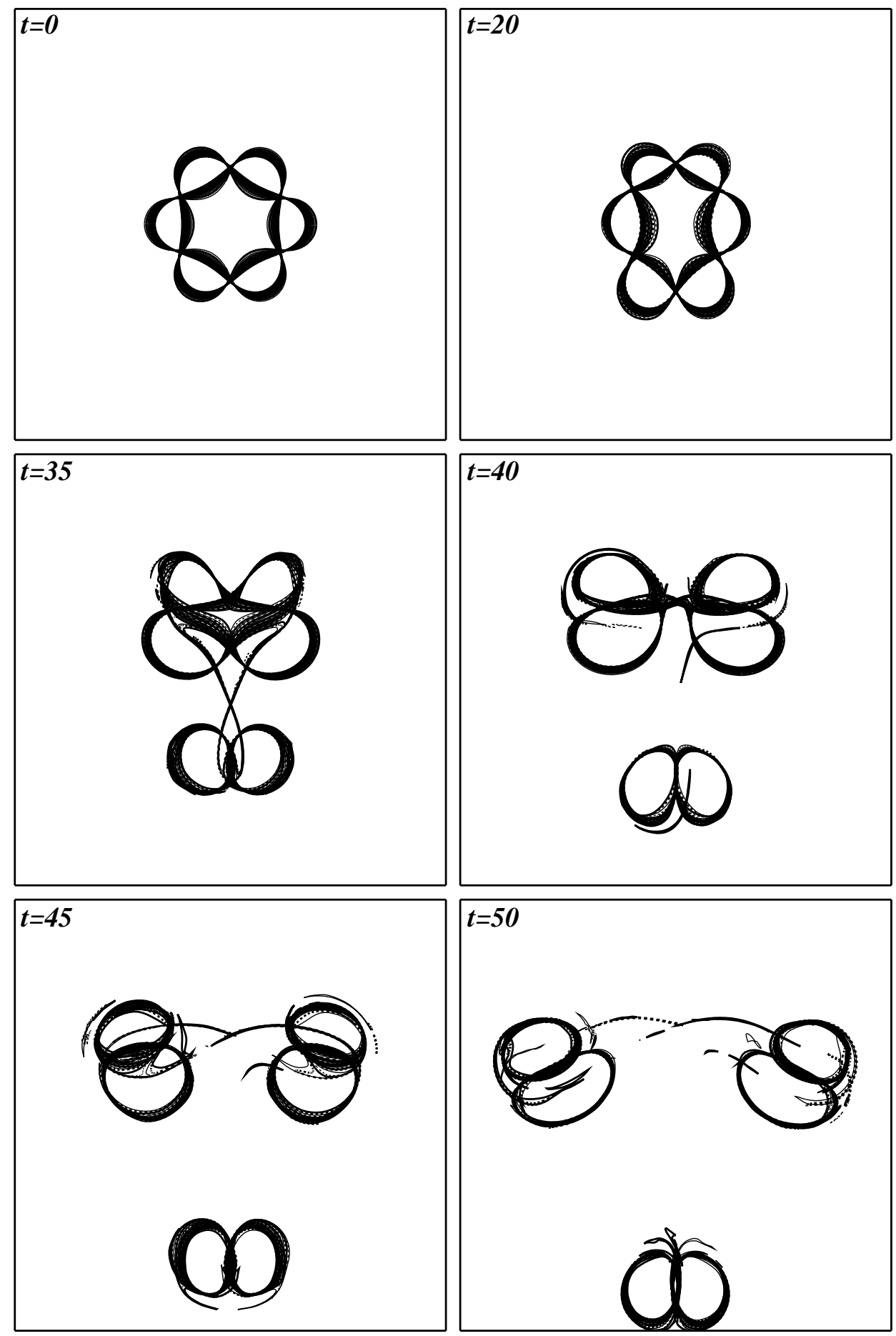

Figure 17. Nonlinear simulation of the last equilibrium state presented figure 16 . $r / h_{v}=5.5, d / h_{v}=1$ and $\Delta x$ small. 


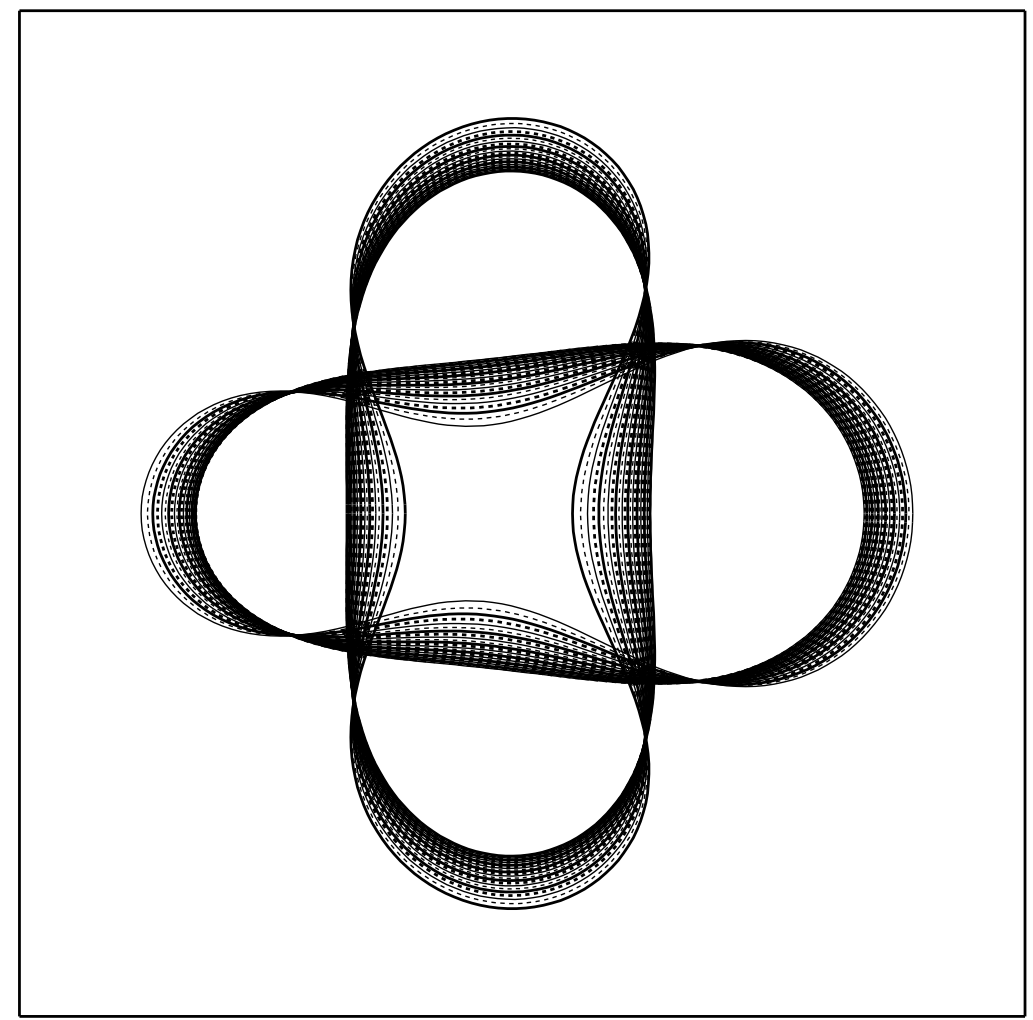

Figure 18. Illustration of the possibility of mode 2-like branch of equilibria for $r / h_{v}=3.5$ and $d / h_{v}=1$.

is height dependent. Secondly the shape of the deformation mode in the vertical has an impact on the growth rate of the modes. It also has an impact on the margin of stability of the modes.

Finally we have also illustrated the existence of other branches of solutions for the equilibria. These equilibria have different shapes and stability properties compared to the first branch investigated. These branches are connected to the emergence of instability modes. These equilibria do not stem from cylindrical vertically aligned vortices. However, we conjecture that they may come from the same branch at $\infty$. The different branches may be separating as the horizontal distance between the poles is decreased. Further work, beyond the scope of this research would be necessary to fully investigate such secondary branches. One possibility would be start the investigation with a guess for the equilibria close to the targeted branch.

\section{Appendix}

Details about the stability analysis are provided below. It is based upon the analysis of small deformations to the contours defining the vortices, and originates from twodimensional version by Polvani and Dritschel (1993). The perturbed boundary of a 
contour $\mathcal{C}_{k}$ is written as:

$$
\boldsymbol{\rho}_{k}\left(\phi_{k}, t\right)=\boldsymbol{\rho}_{e, k}\left(\phi_{k}\right)+\gamma_{k}\left(\phi_{k}, t\right) \frac{\left\{\mathrm{d} y_{e, k} / \mathrm{d} \phi_{k},-\mathrm{d} x_{e, k} / \mathrm{d} \phi_{k}\right\}}{\left(\mathrm{d} x_{e, k} / \mathrm{d} \phi_{k}\right)^{2}+\left(\mathrm{d} y_{e, k} / \mathrm{d} \phi_{k}\right)^{2}}
$$

where $\phi_{k}$ is the 'travel time coordinate', i.e. a quantity proportional to the time that a fluid particle takes to travel along the contour $\mathcal{C}_{k} \cdot \boldsymbol{\rho}_{e, k} \equiv\left(x_{e, k}, y_{e, k}\right)$ is the horizontal position vector describing the equilibrium, and $\gamma_{k}$ is the perturbation in the normal direction to the contour $\mathcal{C}_{k}$. The perturbation equation reads

$$
\frac{\partial \gamma_{k}}{\partial t}+\Omega_{e, k} \frac{\partial \gamma_{k}}{\partial \phi_{k}}=-\sum_{l=1}^{n_{c}} q_{l} \frac{\partial}{\partial \phi_{k}} \oint_{\mathcal{C}_{l}} \gamma_{l} G_{k, l}(\varrho) \mathrm{d} \phi_{l}^{\prime}
$$

where $G_{k, l}(\varrho)$ is the Green's function giving the velocity induced in the layer containing the contour $\mathcal{C}_{k}$ by the PV within the contour $\mathcal{C}_{l}, q_{l}$ is the PV jump across $\mathcal{C}_{l}, \Omega_{e, k}$ is the constant rotation rate of fluid particles along $\mathcal{C}_{k}, \varrho=\left|\boldsymbol{\rho}_{e, k}\left(\phi_{k}\right)-\boldsymbol{\rho}_{e, l}\left(\phi_{l}^{\prime}\right)\right|$ denotes the horizontal distance between the contour points, and $n_{c}$ denotes the total number of contours.

The perturbation function $\gamma_{k}$ is expanded in a truncated Fourier series expansion as:

$$
\gamma_{k}\left(\phi_{k}, t\right)=e^{\sigma t} \sum_{m=1}^{M} A_{k, m} \cos \left(m \phi_{k}\right)+B_{k, m} \sin \left(m \phi_{k}\right)
$$

Here, we use $M=10$ azimuthal modes. $m=1$ may represent the displacement of a contour, hence enable to capture tilting modes.

\section{References}

Ebbesmeyer C. C., Taft B. A., McWilliams J. C., Shen C. Y., Riser S. C., Rossby H. T., Biscaye P. E. and Östlund H. G. 1986 Detection, Structure and Origin of Extreme Anomalies in a Western Atlantic Oceanographic Section J. Phys. Ocean. 16 591-612.

Robinson 1983 Eddies in Marine Science. Springer Verlag.

Vallis, G.K. 2006 Atmospheric and Oceanic Fluid Dynamics. Fundamentals and Large-scale Circulation. Cambridge University Press

von Hardenberg J. McWilliams, J. C., Provenzale A. Shchpetkin A. and Weiss, J. B. 2000 Vortex merging in quasi-geostrophic flows, J. Fluid Mech. 412 331-353

Hogg, N. G., Stommel, H. M. 1985 The heron, an elementary interaction between discrete baroclinic geostrophic vortices and its implications concerning eddy heat flow. Proc. R. Soc. Lond. A. 397 $1-20$.

Dritschel D. G. 2002 Vortex merger in rototating stratified flows J. Fluid Mech. 455 83-101

Reinaud J. N. and Dritschel D. G. 2002 The merger of vertically offset quasi-geostrophic vortices J. Fluid Mech. 469 287-315

Bambrey R. R., Reinaud J. N. and Dritschel D. G. 2007 Strong interactions between two co-rotating quasi-geostrophic vortices J. Fluid Mech. 592 117-133

Ozugurlu E., Reinaud J. N.and Dritschel D. G. 2008 Interaction between two quasi-geostrophic vortices of unequal potential vorticity J. Fluid Mech. 597 395-414

Reinaud J. N. and Dritschel D. G. 2009 The destructive interactions between two counter-rotating quasi-geostrophic vortices J. Fluid Mech 639 195-211 
Reinaud J. N. and Carton X. 2009 The stability and the nonlinear evolution of quasi-geostrophic hetons J. Fluid Mech 636 109-135

Flierl G. 1988 On the stability of geostrophic vortices J. Fluid Mech 197 339-388.

Sokolovskiy M. 1997 Stability of an axisymmetric three-layer vortex Izvestiya, Atmospheric Oceanic Phys. 33 16-26.

Pierrehumbert R. T. 1980 A family of steady, translating vortex pairs with disturbed vorticity. J. Fluid Mech 99 129-144.

Gryanik, V. M. (1983a) Dynamics of localized vortex perturbations vortex charges in a baroclinic fluid, Izvestiya atmospheric and oceanic physics 19 347-352.

Gryanik, V. M. (1983b) Dynamics of singular geostrophic vortices in a two-layer model of the atmosphere (ocean), Izvestiya atmospheric and oceanic physics 19 171-179.

Young, W. R. (1985). Some interactions between small numbers of baroclinic, geostrophic vortices. Geo. and Astro. Fluid Dyn 33 35-61.

Gryanik, V. M. and Tevs, M. V. (1989) Dynamics of singular geostrophical vortices in a N-lavel model of the atmosphere (ocean) Izvestiya atmospheric and oceanic physics 25 179-188.

Griffith R. W., Hopfinger E. J. (1986) Experiments with baroclinic vortex pairs in a ro- tating fluid. J. Fluid Mech 173 501-518

Gryanik, V. M., Sokolovskiy, M. A. and Verron, J. (2006), Dynamics of heton-like vortices. Regular and Chaotic Dynamics, 11(3), 383-434

Sokolovskiy, M. A. and Verron, J. (2014), Dynamics of Vortex Structures in a Stratified Rotating Fluid Springer, 382 pp.

Dritschel, D. G., Reinaud,J. .N. and McKiver, W. J. (2004) The quasi-geostrophci ellipsoidal model J. Fluid Mech. 505, 201-223.

Dristchel, D. G., Scott, R. K. and Reinaud, J. N. (2005) The stability of quasi-geostrophic ellipsoidal vortices J. Fluid. Mech 536, 401-421.

Gryanik, V. M., Doronina, T. N., Olbers, D. and Warncke, T. H. (2000) The theory of three-dimensional hetons and vortex-dominated spreading in localized turbulent convection in a fast rotating stratified fluid. J. Fluid Mech. 423, 71-125. 